\title{
Imaging features of a rare case of scapuloiliac dysostosis (Kosenow syndrome) in an ambulatory child
}

\author{
John Joseph, MB BS \\ Harish Narayanan, MB BS, DMRD \\ Jacob Varghese, $M B B S, M D$ \\ Harish Babu, MB BS, DMRD \\ A Praveen, MB BS
}

Department of Radiodiagnosis, Trivandrum Medical College, Kerala, India

\section{Introduction}

Scapuloiliac dysostosis, also known as pelvis-shoulder dysplasia and Kosenow syndrome, is a rare skeletal dysplasia described first by Kosenow et al. in 1970. ${ }^{1}$ The main components of the syndrome are varying degrees of bilateral iliac and scapular hypoplasia. Various other skeletal abnormalities including exaggerated lumbar lordosis, and clavicular, vertebral, rib and long bone anomalies and extra-skeletal

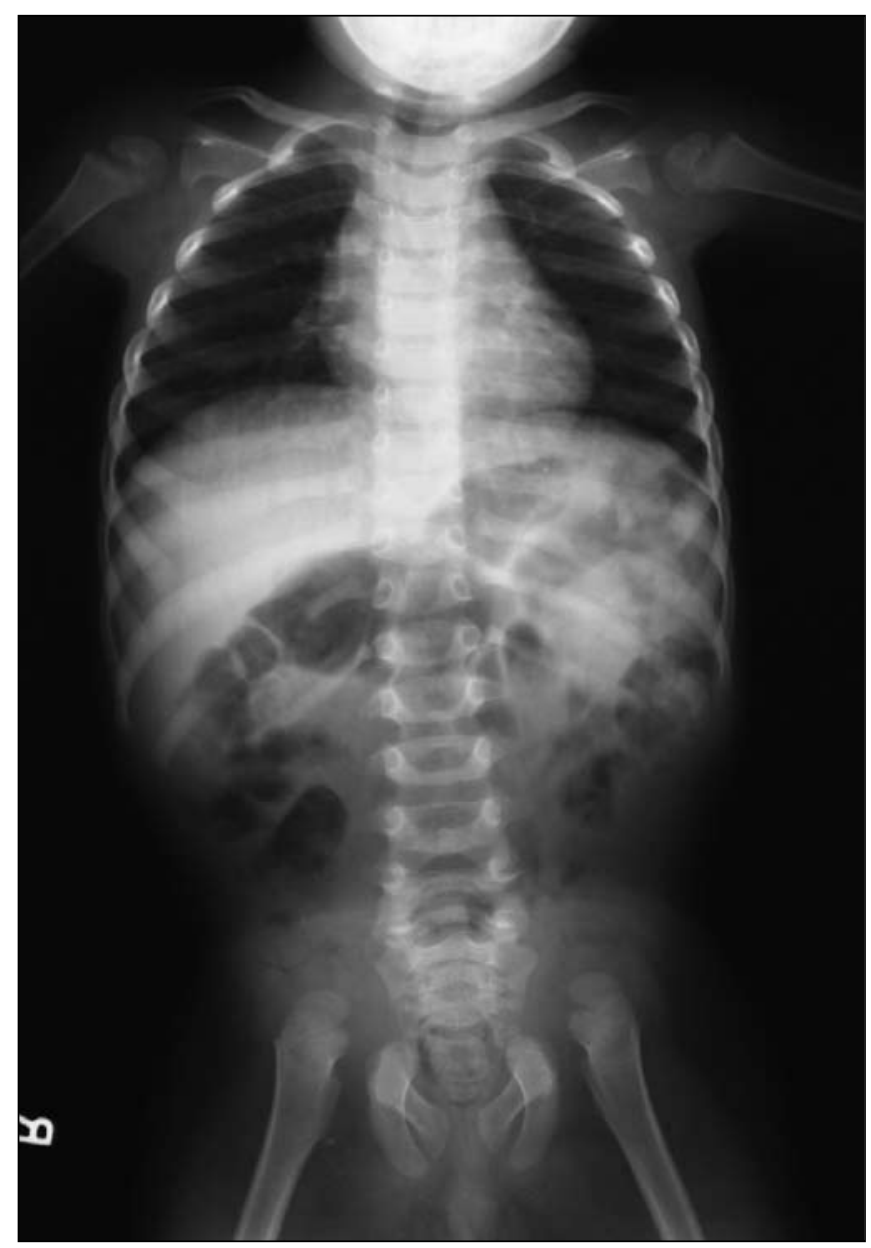

Fig. 1. Frontal radiograph of chest and abdomen shows bilateral absence of ilium and severe hypoplasia of the body of scapulae. The coracoid and acromion are normal bilaterally.

abnormalities, most commonly those of eye and ear, have also been reported. ${ }^{2}$ We report the plain radiography, CT and MRI findings of an interesting case of the syndrome.

\section{Case report}

A 4-year-old boy, the second born of non-consanguinous marriage, presented with abnormal gait; he had a normal neurological examination except for the waddling gait. The elder sibling was normal. Plain radiography of the pelvis showed complete bilateral absence of the ilium, with normal well-formed ischium, the superior and inferior pubic rami, as well as the femoral heads bilaterally. Frontal chest radiograph revealed severe hypoplasia of the body of scapulae bilaterally while the acromion, coracoid and glenoid were well formed. The shoulder joint was normally aligned.

CT scan of the pelvis confirmed the radiographic findings. Band-like soft-tissue density areas were seen in the normal location of the ilium, and the femoral heads were seen lying within the soft tissues. Sacral spina bifida was also noted.

MRI was performed and showed absence of osseous or cartilaginous tissue in the normal location of the ilium. Instead there was a soft-tissue structure, hypo-intense in all sequences, suggestive of fibrous tissue

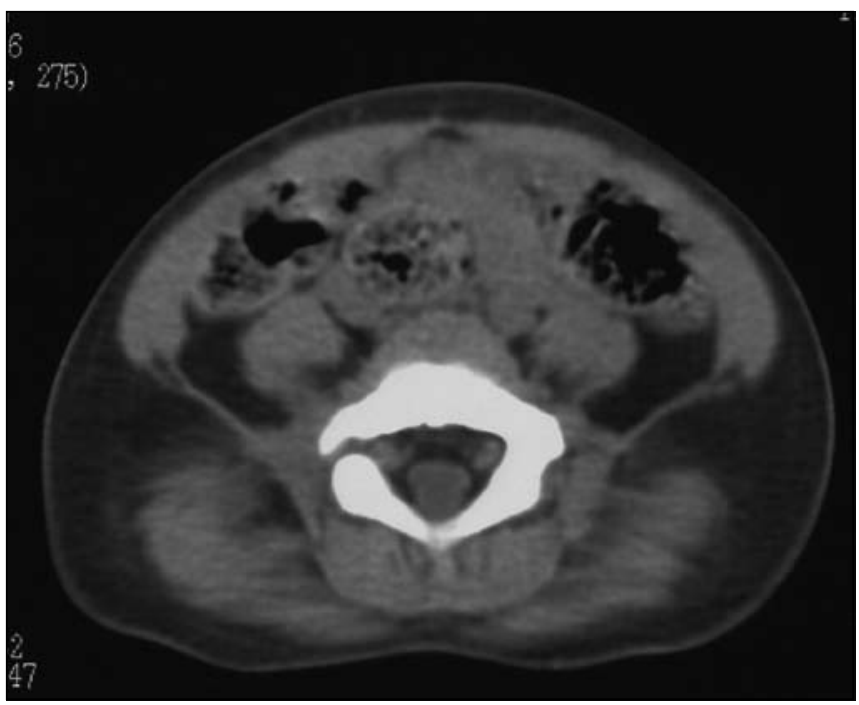

Fig. 2. Axial CT section of pelvis in soft-tissue window setting shows bilateral absence of bony ilia replaced by band-shaped soft-tissue density structures. 


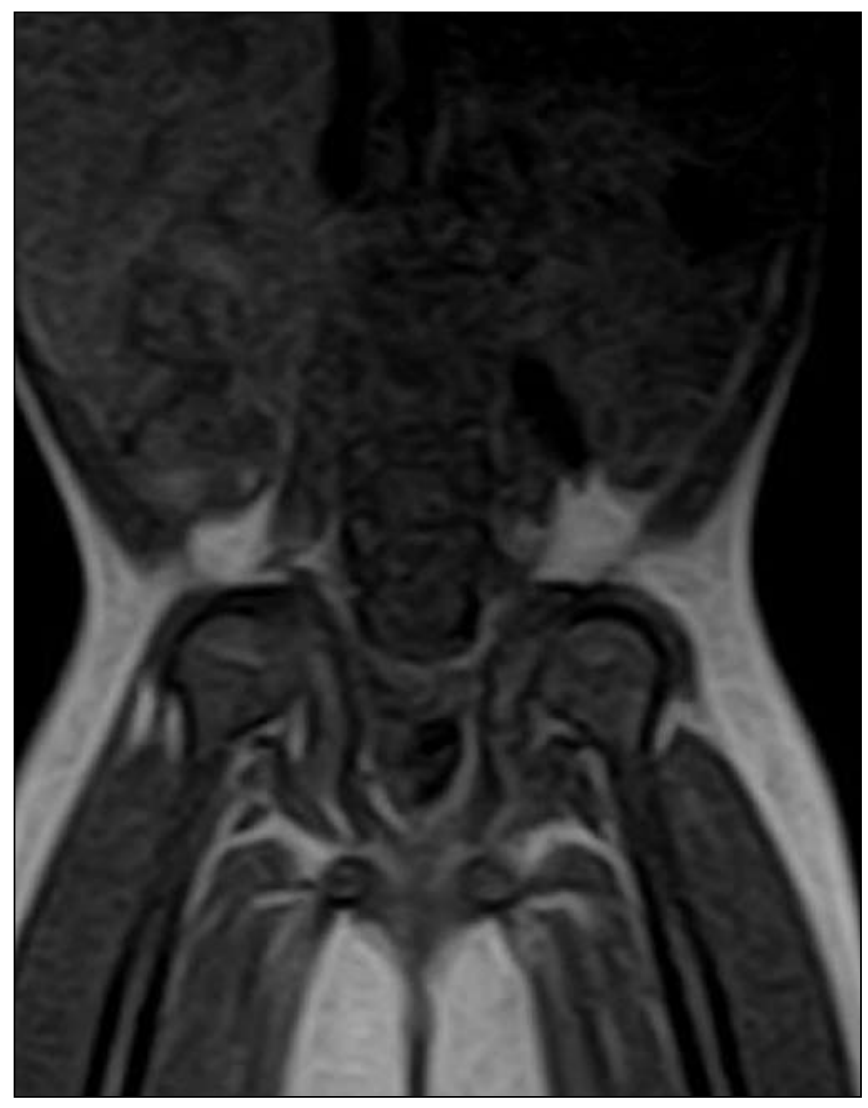

Fig.3. Coronal T1 pelvic MRI reveals iliac agenesis with the femoral heads normal and supported by surrounding muscles and fibrous tissue.

which, along with the surrounding skeletal muscles, seemed to be supporting the well-formed femoral heads.

\section{Discussion}

Scapulo-iliac dysostosis (pelvis-shoulder dysplasia, Kosenow syndrome) is an uncommon hereditary skeletal dysplasia. It is classified as autosomal dominant owing to one of the original families, though both dominant and recessive inheritances are described. ${ }^{3}$ The chief components of the syndrome are varying degrees of bilateral hypoplasia of the ilium and scapulae, usually severe. The sacrum, ischium and pubis are normal while the acetabulum is markedly dysplastic with severe hip instability. The acromion and coracoid process of the scapula are usually preserved despite severe hypoplasia of the body. Milder phenotypical variants may have involvement limited to the pelvis. Several other skeletal and extraskeletal anomalies have also been described; these include hypoplastic or abnormally elongated clavicles, lumbar hyperlordosis, rounded appearance of the vertebral bodies, rib anomalies and overconstriction of the femora and tibiae. ${ }^{2,3}$ The common soft-tissue associations involve the eye and ear e.g. micro-ophthalmia, coloboma of the lids, low-set ears and deafness. ${ }^{4}$

A unique feature of the case under discussion is the well-formed femoral heads and ambulation with a fully functional though abnormal gait, despite the complete bilateral iliac agenesis. To the author's knowledge, only one case of this syndrome with complete iliac agenesis and complete ambulation has been reported whereas the others had severe hip instability and were bedridden. ${ }^{5}$ The support for the femoral head, in the absence of the ilium, seems to be provided by soft tissues such as the surrounding muscles and the fibrous tissue replacing the ilium, as shown by MRI. This seems to be the first report of MRI findings of this syndrome.

The case further supports the theory that the dysplastic changes in the femoral head in congenital dislocation of the hip are a result of its abnormal contact with the ilium or false acetabulum, and that the femoral head will grow normally even if dislocated, in the absence of this abnormal contact. ${ }^{5}$

This case report is believed to be unique in terms of rarity as well as the complete multi-modality radiological evaluation of the syndrome. In addition, the surprising degree of ambulation and well-formed femoral heads despite complete iliac agenesis provide insights into the development of the hip and femoral head.

1. Kosenow W, Niederle J, Sinios A. Becken-schulter dysplasie. Fortschr Geb Rontgenstr Nuklearmed 1970;113:36-48.

2. Taybi H, Lachman R. Radiology of syndromes, metabolic disorders, and skeletal dysplasias, 4th ed. St Louis: Mosby Year Book, 1996: 444, 773, 789.

3. Elliot AM, Roeder ER, Witt DR, et al. Scapuloiliac dysostosis (Kosenow syndrome, pelvis-shoulder dysplasia) spectrum: Three additional cases. Am J Med Genet 2000;95:496-506.

4. Blane CE, Holt JF, Vine AK. Scapuloiliac dysostosis. Br J Radiol 1984;57:526-528

5. Mac-Thiong JM, Leduc S, Labelle H. Complete bilateral agenesis of the ilium in a 7-year-old ambulatory girl. Spine 2005;30:E420-E423 